\title{
An Applied Component Modeling to the Irreversibility from a New Configurationally Perspective of the StatisticalPhysics
}

\author{
Saeed Shahsavari ${ }^{1}$, Mehran Moradi ${ }^{2}$ \\ ${ }^{1}$ Isfahan University of Technology \\ Isfahan, Iran \\ Email: s.shahsavari [AT] me.iut.ac.ir \\ ${ }^{2}$ Isfahan University of Technology \\ Isfahan, Iran \\ Email: moradi [AT] cc.iut.ac.ir
}

\begin{abstract}
From the perspective of statistical physics (Boltzmann equation), configurational entropy can be calculated using the study of the microstates of the system. When a physicalprocess isperformed, identifying the entropy production can be used to investigate the irreversibility, but from the perspective of the Boltzmann equation, to study entropy production, both all microstates and macrostates must be studied. Therefore, a very large volume of calculations will be needed. In this report, using a new innovative energy structure equation, a new macroscopic component modeling is extracted to investigate the configurational irreversibility. To investigate the irreversibility in physical systems, the energy structure equation of the system can be studied in different paths. During performing a physical process, some activated energy components related to the reversible process and remain willbe related to the irreversible process. In this report, also using a quasi-statistical approach, the structure of irreversible components is studied. When macroscopic energy components are the base of the equations, a very large volume of the needed calculations will be less than Boltzmann equation and in fact, studying all particles isn't needed, but it is enough that a few macroscopic components to be investigated. Also, considering the theories of dissipated energy, the extracted equations have the same base as the differentformulations of the second law of thermodynamics.
\end{abstract}

Keywords - Thermodynamics; Statistical physics; Configurational entropy; generated entropy; Energy structure; Energy components; Applied modeling; Irreversibility components

\section{INTRODUCTION}

The only physical law that considers the concept of direction of physical processes is the second law of thermodynamics [1]. In fact, this law introduces reversible and irreversible processes [2]. In the classical form, the second law of thermodynamics has been presented as follows [3]:

$\oint \frac{\delta Q}{T} \leq 0$

Where $\delta Q$ is the heat exchanged at temperature $T$.

One of the other formulation for this law is: $d s_{\text {gen }} \geq 0$, which $s_{\text {gen }}$ is the generated entropy. This statement take this concept that irreversibility is one of the most important quality on the physicalprocesses.

Also, to study the irreversibility fromthe classical thermodynamic point of view, equation (2) can be known as one of the most important relevant equations [4]:

$\overrightarrow{I_{k}}=\sum_{l} L_{k l} \vec{F}_{l}$

Where $\vec{I}_{k}$ is a set of general currents and $\vec{F}_{l}$ is thermodynamic forces. $L_{k l}$ is also a matrix of kinematic coefficients. . Also, the $L_{k l}$ matrix is required to satisfy the symmetry equations in the OSAGER equations [5].

The second law of thermodynamics is the most important physical law that must be applied to all performed processes $[1,6]$. This physical law defines the physical quantity as entropy. According to this law, the entropy of an is olated system will be increased always [1]. However, in the material structures point of view, increasing entropy increases irregulanity [3]. Also, from statistical mechanics perspective, increasing entropy means increases the probability [7], and from a statis tics quantum point of view, it means increase the number of energy levels [8]. In fact, increasing generated entropy 
defines the physical direction of a physical process and must be established in all performed processes. Of course, some cases pose challenges to the second law [9].

Entropy is the quantity that is defined by the second law of thermodynamics. This quantity is extracted to different problems and has different types. One of the most important types of entropy is configurational entropy [10]. There are several methods to investigate configurational entropy $[11,12]$. In some of the materials this type of entropy is the most important type of entropy that must be investigated [13,14]. Of course, because of the exiting methods need to study all sub-structures of the system, calculating the configurational entropy needs a large volume of calculations $[14,15]$.

In this report, using the macroscopic energy components of the system, the energy structure is extracted, and based on it, the irreversibility is investig ated using a new macroscopic quasi-statistical approach. Finally, new macroscopic modeling of the irreversibility components is extracted.

\section{ENTROPY FROM THE PERSPECTIVE OF THE QUANTUUM-STATISTICAL PHYSICS}

From the pers pective of the statistical physics, as studying entropy, all particles must be studied. Also, based on the Boltzmann equation, entropy is considered as a function of the probability [16,17]:

$S=f(W)$

Where $S$ is entropy and $W$ is the probability.

Due to the expected properties to the entropy:

$S=S_{1}+S_{2}$

$f\left(\left(W_{1} \cdot W_{2}\right)=f\left(W_{1}\right)+f\left(W_{2}\right)\right.$

Therefore:

$S=k \ln (W)+$ constant

That $k$ is a universal constant. Equation (6) takes the general dependence between the absolute value of the entropy and probability. There are several equations, using the quantum concepts, for $W$ as a function of the number and weighting factor of the energy levels of the particles [18]. For example, equation (7) states Fermi-Dirac equation of $W$ :

$W=\prod_{j} \frac{g_{j} !}{\left(g_{j}-1\right) ! N_{j} !}$

That $N_{j}$ is the number of particles with the weighting factor of energy level $g_{j}$.

To investigate irreversibility in physical processes from the point of view of the statistical physics, non-equilibrium statis tical operator method can be used [8,17].

\section{ENERGY STRUCTURE MODELING}

The dis sipation of the energy can be investigated by studying the dynamics of the particles [19-22]. One of the most important results that can be concluded from these references, is that when a physical process is performed, due to the condition of the energy applying, some dependent energy components can be activated. Therefore, equation (8) can be presented innovatively as the energy structure equation of the system, with a quasi-statistical base:

$U_{T}=\left(u_{i}\right)+\left[g_{i}\right]+\left[h_{i}\right]+U_{T_{0}}$

Where:

$g_{i}=g_{i}\left(u_{i}\right)$

$h_{i}=h_{i}\left(\dot{u}_{i}\right)$

That functions $g_{i}$ and $h_{i}$ are dependentenergy components activated in the performed physical process.

Using the different formulations of the second law, it can be concluded that equation (8) has the same base as these formulations [23]. 


\section{IRREVERSIBILITY ANALYSIS AND COMPONENT MODELING}

To irreversibility analysis, it is supposed that some energy is applied to the system with different conditions [4]. In fact, the systemcan experience different paths. In this case, we use a quasi-static path $\left(\dot{u}_{i}=0\right)$ as the reference to study general paths $\left(\dot{u}_{i} \neq 0\right)$.

Therefore, it should be assumed that the component $u_{i}$ is an independent component activated in the performed process, as can be seen in figure 1 .

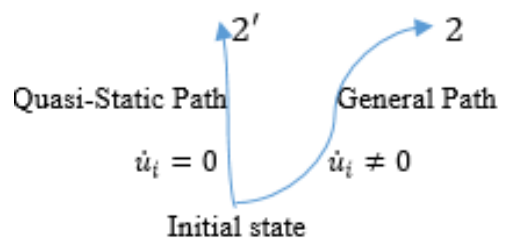

Figure 1. Scheme of the performed process

Variation of the equation (12) can be calculated as follows:

$\delta U_{T}=\delta\left(u_{i}+g_{i}+h_{i}+U_{T_{0}}\right)$

If in path $1-2^{\prime}$ the independent component of energy changes to the amount of $\delta u^{\prime}{ }_{i}$, Therefore equation (11) can be written as follows:

$\delta U_{T}=\delta u_{i}^{\prime}+\frac{\partial g_{j}}{\partial u_{i}} \delta u_{i}^{\prime}$

And in path $1-2$ :

$\delta U_{T}=\delta u_{i}+\frac{\partial g_{i}}{\partial u_{i}} \delta u_{i}+\frac{\partial h_{i}}{\partial \dot{u}_{i}} \delta \dot{u}_{i}$

Therefore, equation (14) must be satisfied:

$\left(1+\frac{\partial g_{i}}{\partial u_{i}}\right)\left(\delta u_{i}-\delta u_{i}^{\prime}\right)=-\left(\frac{\partial h_{i}}{\partial \dot{u}_{i}}\right) \delta \dot{u}_{i}$

The value $\left(\delta u_{i}-\delta u_{i}^{\prime}\right)$ can be considered as the variation between quasi-static and general paths, as can be seen in figure 2 .

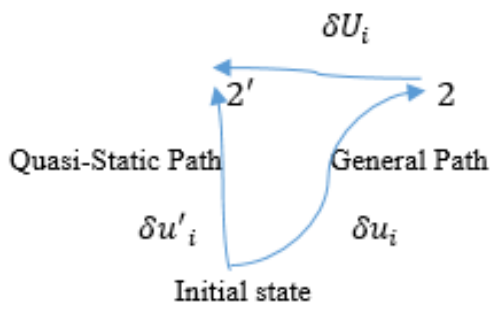

Figure 2. The variation between quasi-static and general paths

Therefore, equation (14) can be written as follows:

$\left(1+\frac{\partial g_{i}}{\partial u_{i}}\right)\left(\delta U_{i}\right)=-\left(\frac{\partial h_{i}}{\partial \dot{u}_{i}}\right) \delta \dot{U}_{i}$

Due to the second law of thermodynamics, $\delta u^{\prime}{ }_{i} \geq \delta u_{i}$ for the conditions $\delta U_{T}>0$ and $\delta u^{\prime}{ }_{i} \leq \delta u_{i}$ for the conditions $\delta U_{T}<0$. Also, because of the function $g_{i}$ will be activated in all possible paths, therefore $\frac{\partial g_{i}}{\partial \lambda_{i}} \geq 0$.Therefore, based on the equation (15), if $\delta U_{T}>0$, it can be concluded $\left(\frac{\partial h_{i}}{\partial \dot{u}_{i}}\right) \delta \dot{U}_{i}>0$ and if $\delta U_{T}<0$, so $\left(\frac{\partial h_{i}}{\partial \dot{u}_{i}}\right) \delta \dot{U}_{i} \partial u_{i}<0$. Also, in the quasi-static path $\left(\dot{u}_{i}=0\right),\left(\frac{\partial h_{i}}{\partial \dot{u}_{i}}\right) \delta \dot{U}_{i}=0$.

And by defining:

$\varphi_{i}=\frac{\left(\frac{\partial h_{i}}{\partial \dot{u}_{i}}\right) \delta \dot{U}_{i}}{\delta U_{T}}$

So $\varphi_{i} \geq 0$ always. Therefore, $\varphi_{i}$ can be used as a quantity to investigate irreversibility in physical processes. Because of $\varphi_{i}$ is a function of the macroscopic energy component, so it depends on the macrostate of the system. Also, Due to the 
kinematic theory of the dissipated energy $[19,22]$, equation(16) has the same base with the different formulations of the second law of thermodynamics.

\section{CONCLUSIONS}

During performing a physical process, identifying entropy production can be used to study the irreversibility. The Boltzmann equation investigates the microstates of the system to calculate the entropy. Also, the Boltzmann equation can be used for physical systems in that particles have no many degrees of freedom, as configurational entropy. From the perspective of statistical physics (Boltzmann equation), configurational entropy can be calculated using the study of the micros tates of the system. Therefore, a large volume of calculations will be needed.

From the perspective of the energy structure, one of the most important results can be concluded from the theories of dis sipated energy, using the macroscopic energy component, is formulated by equation (8). This innovative formulation has the same base as different formulations of the second law of thermodynamics and can be used to study the irreversibility without the need to investig ate all sub-structures.

To study irreversibility, the energy structure equation is investigated in different paths. In fact, it is assumed that some equal energy is applied to the system in different conditions, then the variation of the energy structure equation between these paths is examined. Finally, equation (16) takes a physical quantity to study the irreversibility $\left(\varphi_{i} \geq 0\right)$. Because the quantity $\varphi_{i}$ depends on the macros tates of the system, it needs very little calculation than investigating all sub-structures of the system.

\section{REFERENCES}

[1] Sheehan, D. P. "The second law of thermodynamics: Foundations and status." Foundations of Physics 37.12 (2007): 1653-1658.

[2] Erlichson, Herman. "Sadi Carnot,Founder of the Second Law of Thermodynamics'." European journal ofphysics 20.3 (1999): 183.

[3] Carnot, Sadi, RudolfClausius, and William Thoms on Baron Kelvin. The Second Law of Thermodynamics. American Book Company, 1899.

[4] Luzzi, Roberto, Áurea R. Vasconcellos, and J. Galvão Ramos. "On the statistical foundations of irreversible thermodynamics." Fortschritte der Physik: Progress of Physics 47.4(1999): 401-432.

[5] Ons ager, Reciprocal relations in irreversible processes I. Phys. Rev. 37, 405 (1931); ibid. II 38, 2235 (1931).

[6] Kostic, Milivoje M. "Revisiting the second law of energy degradation and entropy generation: From Sadi Carnot's ingenious reasoning to Holistic generalization." AIP Conference Proceedings. Vol. 1411. No. 1. American Institute of Physics, 2011.

[7] Goldstein, Sheldon. "Boltzmann's approach to statistical mechanics." Chance in physics. Springer, Berlin, Heidelberg, 2001. 39-54.

$\lceil 8\rceil$ Wallace, David. "Probability and irreversibility in modern statistical mechanics: Classical and quantum." Quantum Foundations of Statistical Mechanics ( Oxford University Press, forthcoming) (2016).

[9] Č́pek, V., and D. P. Sheehan. "Quantum mechanical model of a plas ma sy stem: a challenge to the second law of thermod ynamics." Physica A: Statistical Mechanics and its Applications 304.3-4 (2002): 461-479.

[10] Lambert, Frank L. "Configurational entropy revisited." Journal of Chemical Education 84.9 (2007): 1548.

[11] Karplus, Martin, and Joseph N. Kushick. "Method for estimating the configurational entropy of macromolecules." Macromolecules 14.2(1981): 325-332.

[12] Di Marzio, Edmund A., and Arthur JM Yang. "Configurational entropy approach to the kinetics of glas ses." Journal of research of the National Institute of Standards and Technology 102.2(1997): 135.

[13] Richet, Pascal. "Viscosity and configurational entropy of silicate melts." Geochimica et Cosmochimica Acta 48.3 (1984): 471-483.

[14] Richert, Ranko, and C. A. Angell. "Dynamics of glas s-forming liquids. V. On the link between molecular dynamics and configurational entropy." The Journal of chemical physics 108.21 (1998): 9016-9026.

[15] Harpole, Kyle W., and Kim A. Sharp. "Calculation of configurational entropy with a Boltzmann-quasiharmonic model: the origin of high-affinity protein-lig and binding." The Journal of Physical Chemistry B 115.30 (2011): 9461-9472.

[16] Coveney, Peter V. "The second law of thermodynamics: entropy, irreversibility and dynamics." Nature 333.6172 (1988): 409-415.

[17] Lucia, Umberto. "Statis tical approach of the irreversible entropy variation." Physica A: Statistical Mechanics and its Applications 387.14 (2008): 3454-3460.

「18] Cohen, E. G. D. "Boltzmann and Einstein: Statis tics and dynamics - an unsolved problem." Pramana 64.5 (2005): 635-643. 
[19] Thomson, William. "9. The kinetic theory of the dissipation of energy." Proceedings of the Royal Society of Edinburgh 8(1875): 325-334.

「201 Burbury, S. H. "LII. A theorem on the dissipation of energy." The London, Edinburgh, and Dublin Philosophical Magazine and Journal of Science 13.83 (1882): 417-419.

[21] Rayleigh, Lord. "On the dissipation of energy." Van Nostrand's Eclectic Engineering Magazine (1869-1879) 12.78 (1875): 519.

[22] Thoms on, William. "2. On a Univers al Tendency in Nature to the Dis sipation of MechanicalEnergy." Proceedings of the Roval Society of Edinburgh 3 (1857): 139-142.

[23] Eu, Byung C. "Kinetic theory and irreversible thermodynamics." NASA STI/Recon Technical Report A 93 (1992). 